\title{
Performance Evaluation of Barker Codes using New Pulse Compression Technique
}

\author{
B. Kiranmai \\ Gitam Institute of Technology \\ Department of ECE \\ Visakhapatnam, India
}

\author{
P. Rajesh Kumar \\ Andhra University \\ Department of ECE \\ Visakhapatnam, India
}

\begin{abstract}
Pulse compression is used to achieve large radiated energy but simultaneously a short pulse for range resolution. Range resolution is an ability of the receiver to detect nearby targets. Pulse compression can also detect small target signals out of clutter. The matched filter output consists of unwanted but unavoidable side lobes however the reduction of this side lobe strength is the active research topic in radar signal processing. In this paper a new technique is proposed to suppress the side lobes of radar signals that result from standard matched filtering. In this technique, it produces better peak side lobe ratio than all other conventional side lobe reduction techniques. In simulation results, the performance of this filter technique for Barker codes is compared with the amplitude shift code side lobe reduction technique.
\end{abstract}

\section{Index Terms:}

Barker code, Peak side lobe Ratio, Wiener filter, right shift code.

\section{INTRODUCTION}

Pulse compression using frequency or phase modulation, allows radar to simultaneously achieve the energy of a long pulse and also the resolution of a short pulse with increased range resolution $[1,6]$. When a target echo signal passes through a matched filter, this filter output however consists of a spike-like main lobe and also some unwanted but unavoidable noise-like side lobes. These side lobes can form spurious targets or mask the main lobe of weak target echo signals at neighboring range cells. But to prevent these problems, we need to design a binary code whose auto correlation function main lobe-to-peak-side lobe ratio is maximized for a given code length. Enormous efforts have been dedicated to the design of a good auto correlation function property for binary sequences [2, 3, 4]. Furthermore, one has to employ a side lobe reduction filter to achieve an adequate main lobe-to-peak-side lobe ratio. The first method is by using a matched filter to perform the pulse compression correlation and this output of the matched filter is cascaded with the mismatched filter to suppress the side lobes. In this contest Rihaczek and Golden (R-G) introduced the R-G filter to suppress the side lobes for Barker codes [2]. But the method suggested in the [2] is not applicable to the binary coded waveforms having negative side lobes and also not supported with optimization. So the other way is to design a mis matched filters directly instead of using weighted function after the matched filter [7]. In this paper the (R-G) filter is designed and that can be used for the Barker codes .The coefficients of this filter are derived by using wiener filter and the results are compared with amplitude shift code pulse compression technique.

\section{BARKER CODED WAVEFORM}

Poly phase coded waveforms are waveforms that use only two phase shifts of 0 and $\pi$.The Phase changes can be used to increase the signal bandwidth of a long pulse for purpose of pulse compression. A long pulse with duration $\mathrm{T}$ is subdivided into sub pulses having width has $t_{b}$.however the bandwidth can be increased by changing the phase of the each sub pulse. If the phase of each sub pulse is 0 or $\pi$ then are termed as binary phase codes. In addition to this if the code that produce compressed waveform with constant side lobe levels equal to unity is the Barker code.

The auto correlation function (which is an approximation for the matched filter output) for code length $N$, Barker code will be $2 \mathrm{Nt}_{\mathrm{b}}$ wide. The main lobe is $2 \mathrm{t}_{\mathrm{b}}$ wide. The peak value is equal to $N$. There are $(N-1) / 2$ side lobes on either side of the main lobe [5].

\section{3. (R-G ) FILTER}

The proposed filter to suppress the side lobes produced at the output of the matched filter is as shown in fig.1

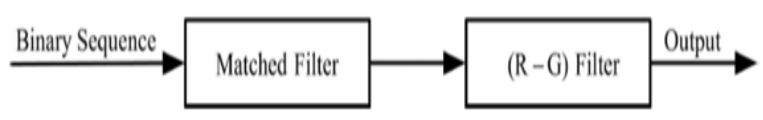

Fig.1

Consider the input wave form at the receiver is $\mathrm{x}(\mathrm{t})$ which possesses the auto correlation function $\mathrm{R}(\mathrm{t})$

$$
\mathrm{R}(\mathrm{t})=\mathrm{x}(\mathrm{t}) * \mathrm{x}(-\mathrm{t})
$$

Where * indicates time convolution [7]. However $\mathrm{R}(\mathrm{t})$ can also be rewritten as two sub functions $(R \mathrm{~m}(\mathrm{t})$ and $R_{s}(t)$ ),representing the contributions of the main lobe and the side lobes respectively. By taking Fourier transform of equation (1) and energy spectrum of that given as

$$
E(f)=E_{m}(f) E_{s}(f) \text {. }
$$

Where $E_{m}(f)=\sin ^{2}(\pi f T) /(\pi f T)^{2}$,

$$
\mathrm{E}_{\mathrm{s}}(\mathrm{f})=\mathrm{N}-1+\sin (2 \pi \mathrm{fNT}) / \sin (2 \pi \mathrm{fT})
$$

and $E_{m}(f)$ and $E_{s}(f)$ represents the spectrum contribution of the main lobe and the side lobes, respectively. It is obvious that to find a network which has a transfer function of $1 / E_{s}(f)$, then the side lobes in every range cell would vanish.

Often it is rather difficult to synthesize a filter with a transfer function exactly equal to $1 / \mathrm{E}_{\mathrm{s}}(\mathrm{f})$. But the closer the transfer function of the filter approximates $1 / \mathrm{E}_{\mathrm{s}}(\mathrm{f})$, the lower the peak output side lobe will be. So the inverse of the $E_{s}(f)$ will be

$H(f)=1 / E_{s}(f)=1 / N-1+\sin (2 \pi f N T) / \sin (2 \pi f T)$ 
Equation(3) can be written as $\mathrm{H}(\mathrm{f})=[\mathrm{N}-1+\sin (2 \pi \mathrm{fNT}) /$ $\sin (2 \pi \mathrm{fT})]^{-1}$. In conclusion the better one who approximates this transfer function, the higher the degree of side lobe suppression.

To analyze filter transfer function $\mathrm{H}(\mathrm{f})$ can be approximated as a geometric series and selecting the first four terms of the $\mathrm{H}(\mathrm{f})$ is given by

\section{$\mathrm{H}(\mathrm{f})=\mathrm{A}+\mathrm{B} \sin (2 \pi \mathrm{fNT}) / \sin (2 \pi \mathrm{fT})+\mathrm{C}[\sin (2 \pi \mathrm{fNT}) / \sin (2 \pi \mathrm{fT})]^{2}+\mathrm{D}$ $[\sin (2 \pi \mathrm{fNT}) / \sin (2 \pi \mathrm{fT})]^{3}$}

where A,B,C,D are coefficients of the (R-G)filter. So the filter transfer function with $\mathrm{m}$ coefficients are called as (R-G-m) stage filter. The impulse response of this $\mathrm{H}(\mathrm{f})$ is given as

$$
\begin{aligned}
& \mathrm{h}(\mathrm{t})=\mathrm{A} \delta(\mathrm{t})+\mathrm{B} \sum_{-(N-1) / 2}^{(\mathrm{N}-1) / 2} \delta(\mathrm{t}-2 \mathrm{nT})+\mathrm{C} \sum_{-(N-1)}^{(\mathrm{N}-1)}(\mathrm{N}- \\
& |\mathrm{n}|) \delta(\mathrm{t}-2 \mathrm{nT}) \ldots \ldots .(4)
\end{aligned}
$$

If the sampled sequence for $h(t)$ is a discrete set $\left\{h_{i}\right\}$. However this discrete set $\left\{h_{i}\right\}$ is cascaded with matched filter out put .So if the discrete auto correlation sequence is $\left\{R_{i}\right\}$ (matched filter out put) for the 13-bit Barker code, the output of the (R-G-2) filter where $m=2$ stage is obtained by

$\left\{y_{i}\right\}=\left\{R_{i}\right\} *\left\{h_{i}\right\}$ where $i=0,1,2$

The output signals of the (R-G-2) filter resulting from convolutions of $\left\{R_{i}\right\}$ with the first term, the second term, the third term of $\left\{h_{i}\right\}$.Wiener filtering method has been used to find the unknown filter coefficient. The Wiener filters are characterized by the following:

1. Assumption: signal and (additive) noise are stationary linear stochastic processes with known spectral characteristics or known autocorrelation and cross-correlation

2. Requirement: the filter must be physically realizable or causal (this requirement can be dropped, resulting in a noncausal solution)

3. Performance criterion: minimum mean-square error (MMSE)

\section{4. (R-G) FILTER WITH WEIGHTING FUNCTION}

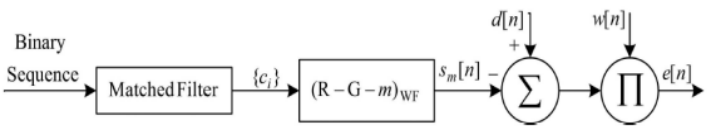

Fig.2

Fig.2 is a diagram of wiener filter with weighting function. The Wiener filter adjusts its weight(s) to produce filter output $s_{m}[n]$, which would be as close as the noise $n(n)$ contained in the output of the matched filter . Hence, at the subtracted output, the noise is canceled, and the output e(n) contains clean signal. Where $d[n]$ is the desired output and $w[n]$ is the weighting function. The error function $\mathrm{e}[\mathrm{n}]$ is the difference of the desired output $d[n]$ to the output of the filter $s[n]$ is multiplied by the weighting function $w[n]$ i.e $e[n]$ $=\mathrm{w}[\mathrm{n}](\mathrm{d}[\mathrm{n}]-\mathrm{s}[\mathrm{n}])$ Now, the wiener filtering technique is used to achieve an optimal filter that minimizes the total energy of $\mathrm{e}[\mathrm{n}]$.

So the method is to minimize the error signal e[n], to get the desired signal with minimum side lobe levels.

The Wiener filtering technique is used to achieve coefficients of the filter, such that it minimizes the total energy of e[n]. So the method is to minimize the error signal e[n] given as MMSE

$$
\mathrm{E}=\sum_{n=-(m+1)(N-1)}^{(m+1)(N-1)}\left(|\mathrm{e}(\mathrm{n})|^{2}\right)
$$

The problem is to find the least value of $\mathrm{E}_{\mathrm{WF}}$. Because of the symmetry of the discrete set, the output $s_{m}[n]$ will be symmetric. Hence, the non-positive part of summation in the equation (6) can be discarded. Also, the desired output $d[n]$ may be desirably set to zero everywhere except in the peak location. In other words d[n] $=\mathrm{N} \delta[n]$. Hence,

$$
\begin{aligned}
& \arg \underset{\mathrm{wk}}{\min } \mathrm{E}_{\mathrm{WF}}=\arg _{\mathrm{wk}} \min \left(\left|\mathrm{w}[0]\left(\mathrm{N}-\mathrm{s}_{\mathrm{m}}[0]\right)\right|^{2}+\right. \\
& \left.\sum_{n=1}^{(m+1)(N-1)}|\mathrm{w}[\mathrm{n}] \mathrm{s}[n]|^{2}\right) \ldots \ldots \ldots \ldots \ldots \ldots \ldots(7)
\end{aligned}
$$

The above equation shows an optimization problem. Hence the problem is to solve the set

$$
\frac{\partial \mathrm{E}_{\mathrm{WF}}}{\partial \mathrm{w}_{\mathrm{k}}}=0 \quad \mathrm{k}=0,1, \ldots \ldots \ldots, . . \mathrm{m} .
$$

The filter coefficients can be normalized so that the main lobe level will be $\mathrm{N}$. the weighting functions can be updated in an iterative manner such that higher side lobes receive greater weights.

Although this optimization reduces the side lobe levels, the reduction may not be uniform over all side lobes.

To alleviate such non uniformity, the weighting functions can be updated in an iterative manner such that higher side lobes receive greater weights. Updating the weights by multiplying them with the current filter outputs gives the higher side lobes greater weights in the next iteration. Therefore, by iterating the algorithm a filter with a relatively flat side lobe level may be derived, which is called the (R-G-m) WWF filter.

1) Set the current weighting function to 1 for all of $n$.

2) Generate an optimal filter using the current weighting function.

3) Add a small constant to the absolute value of all filter outputs.

4) Multiply the current weighting function by the result of step 3 .

5) If uniform side lobes are attained, then stop algorithm. Otherwise, go to step 2

\section{AMPLITUDE SHIFT CODE PULSE COMPRESSION METHOD}

To generate a flat uniform side lobe pattern similar to those obtainable from Barker codes with less complexity in the structure and to reduce the side lobe level a new pulse compression method is proposed. Instead of carrying out subtraction after pulse compression, two identical sequences but shifted by 1-bit can be incorporated into the correlation filter to obtain this magnitude difference curve directly [8]. With this a direct way to achieve ideal unit amplitude side lobes pattern can be implemented. For shifting the code from left to right, right shift registers with D FFs can be used and the block diagram for shifting the Barker code 3 is as shown in figure (3). Where SI is serial input with Barker code 3.

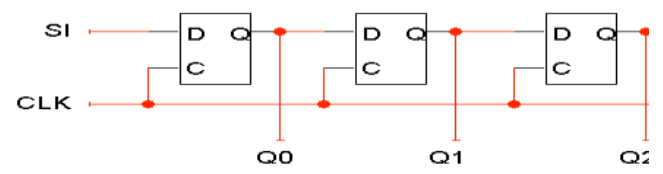

Fig.3 
The block diagram for amplitude shifted code pulse compression technique is as shown in figure (4).

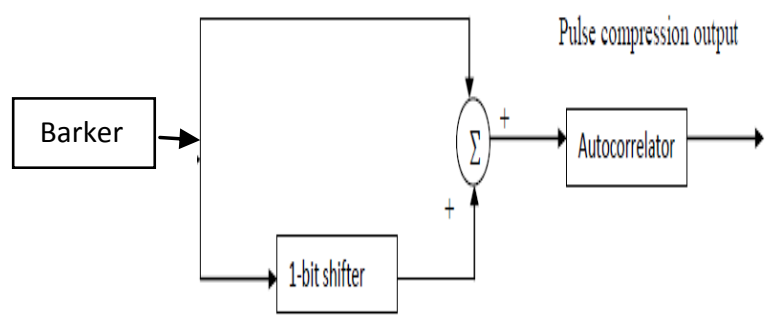

Fig. 4

Where $\mathrm{X}_{\mathrm{i}}$ is Barker code, MF is Matched filter.

In figure 4 the signal is combined with one-bit version of itself and the resulting signal is passed through correlation process.

\section{PERFORMANCE EVALUATION CALCULATIONS FOR BARKER CODE}

Peak side lobe ratio is equal to the ratio of the energy in the chirp corresponding to the highest side lobe normalized by the energy in the chirp at the peak response.

PSLR $=\mid$ Yn peak sidelobe $\left.\right|^{2} /|Y 0|^{2}$

Where $Y_{0}=$ peak voltage response of the filter

Yn $=$ voltage of $n^{\text {th }}$ range side lobe

\section{SIMULATION RESULTS}

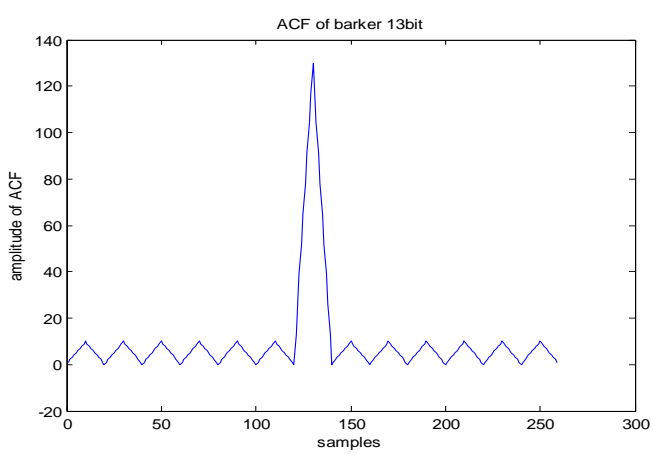

Fig 5.ACF of Barker code 13

Figure 5 shows the autocorrelation function of Barker code length 13

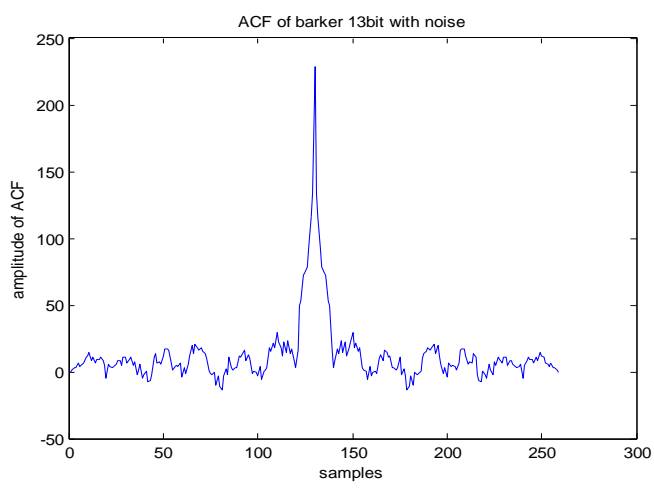

Fig.6 ACF of Barker code with Gaussian noise
Figure 6 is the autocorrelation function of Barker code length 13 with Gaussian noise

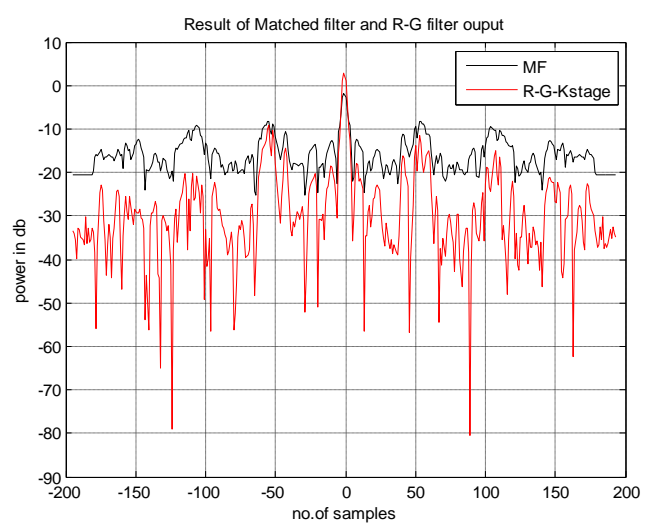

Fig 7. Comparison of MF and R-G-2 stage wiener filter for Barker code 13.

Figure 7 shows the comparison of matched filter with noise and the $\mathrm{R}-\mathrm{G}-\mathrm{m}$ filter for Barker code length is 13 . Where $\mathrm{m}$ is the number of stages in the R-G filter. Here the $m$ value is 2

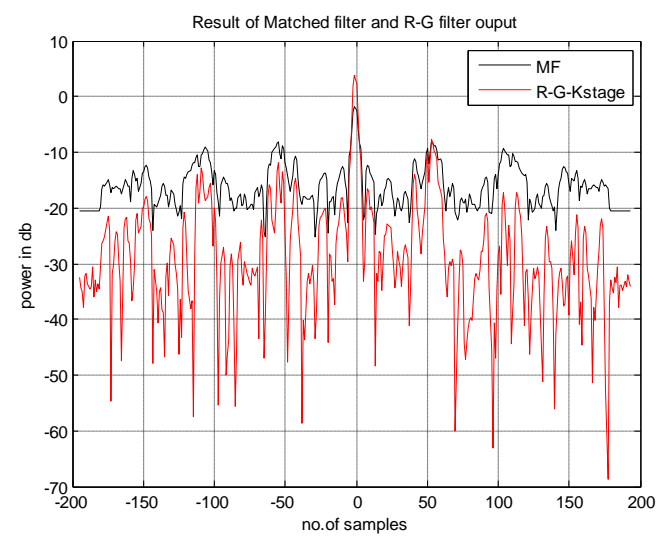

Fig 8. Comparisons of MF and R-G-3 stage wiener filter for Barker code13.

Figure 8 shows the comparison of matched filter with noise and the $\mathrm{R}-\mathrm{G}-\mathrm{m}$ filter for Barker code length is 13 . Where $\mathrm{m}$ is the number of stages in the R-G filter. Here the $m$ value is 3 .

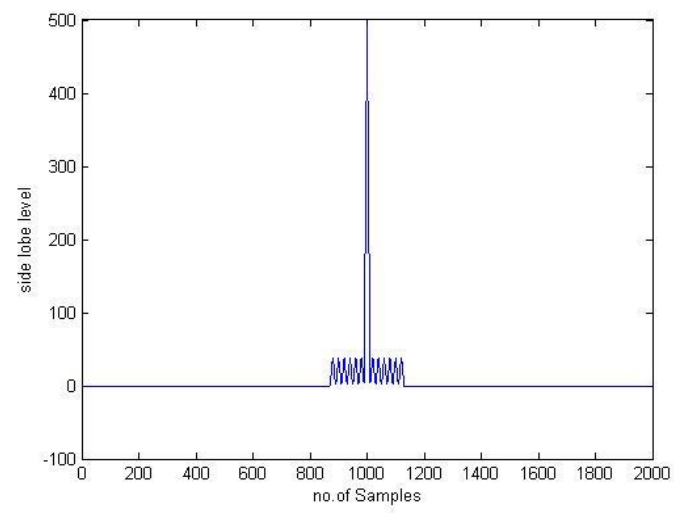

Fig 9. ACF of 1bit shift Barker code length of 13

The signal is combined with one-bit version of itself and the resulting signal is passed through correlation process.

Figure 9 shows the autocorrelation function of the combined signal (itself+ one bit shift) for the Barker code length 13. 


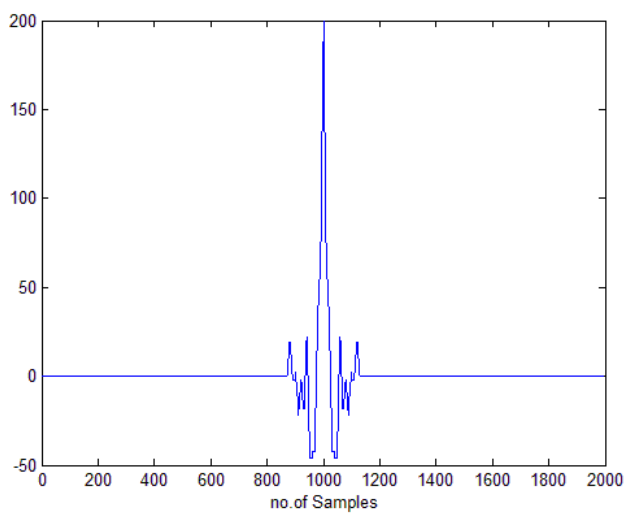

Fig 10. ACF of 2 bit shift Barker code length of 13

The signal is combined with two-bit version of itself and the resulting signal is passed through correlation process.

Figure 10 shows the autocorrelation function of the combined signal (itself +two bit shift) for the Barker code length 13.

Table 1. Comparison between (R-G-m) filter and the one bit shifted Barker code

\begin{tabular}{|c|c|c|c|c|}
\hline $\begin{array}{c}\text { Barker } \\
\text { code }\end{array}$ & $\begin{array}{c}\text { 1-Bit } \\
\text { Shifted } \\
\text { code } \\
\text { PSLR }\end{array}$ & $\begin{array}{c}\text { 2-Bit } \\
\text { Shifted } \\
\text { code } \\
\text { PSLR }\end{array}$ & $\begin{array}{c}\text { (R-G- } \\
\text { 2) } \\
\text { filter } \\
\text { PSLR }\end{array}$ & $\begin{array}{c}\text { (R-G-3) } \\
\text { filter } \\
\text { PSLR }\end{array}$ \\
\hline 5 & 32.18 & 63.37 & 32.68 & 31.61 \\
\hline 7 & 75.83 & 81.7 & 35.81 & 33.09 \\
\hline 11 & 106.7 & 106.8 & 30.31 & 26.76 \\
\hline 13 & 52.62 & 44.14 & 36.18 & 38.3 \\
\hline
\end{tabular}

Table 1 show that the shifted code method have better side lobe level reduction ratio than the(R-G) mismatched filter technique.

\section{CONCLUSIONS}

The new R-G-m filter is observed to be suppresses the side lobes more than that of other techniques. This filter is applicable to any binary coding signals and the filter coefficients can be calculated by the wiener filtering method. But the number of stages is chosen based on the required value of main lobe-to-peak-side lobe level and the LSNR. And also it requires more multipliers and adders depending upon the number of stages increased the hard ware structure also very complicated for higher stages. So the amplitude shifted code method achieves grater side lobe reductions with the simple circuitry and with less number of adders and multipliers. This method can be applied to the combined Barker code.

\section{REFERENCES}

[1] Skolnik, M. I. Introduction to Radar Systems (3rd Ed.). New York: McGraw-Hill, 2001, 339-369.

[2] Rihaczek, A. W. and Golden, R. M. Range side lobe suppression for Barker codes. IEEE Transactions on Aerospace and Electronic Systems, AES-7 (Nov. 1971), 1087-1092.

[3] Baden, J. M. and Cohen, M. N. Optimal side lobe suppression for bi phase codes In Proceedings of the IEEE Tele systems Conference, 1991, 127-131.

[4] Chen Xiao Hua and Juhani Oskman, A New Algorithm to Optimize Barker Code Side lobe and Suppression Filter, IEEE Transactions on Aerospace and Electronic Systems, Vol AES-26, No. 4, July 1990, pp 673-677.

[5] Nadav Levanon, Eli Mozeson, "Radar Signals", John Wiley \& Sons, 2004, Hoboken, New Jersey

[6] Gabriel, W. F. Super resolution techniques in the range domain. In Proceedings of the IEEE International Radar Conference, May 1990, 263-267.

[7] Amirmokhtar Akbaripour Mohammad H. Bastani. Range Side lobe Reduction Filter Design for Binary Coded Pulse Compression System in the publications of the IEEE Log No. T-AES/48/1/943618 .2012 IEEE

[8] A New Pulse Compression Technique for Poly phase Codes in Radar Signals International Symposium on Devices MEMS, Intelligent Systems \& Communication (ISDMISC) 2011 Proceedings published by International Journal of Computer Applications® (IJCA) 